\title{
Fetus in Fetu: A Rare Prenatal Diagnosis of a Fetal Abdominal Mass
}

Ali Ozgur ERSOY ${ }^{*}$, Sibel OZLER ${ }^{1}$, Gulnur GOLLU BAHADIR ${ }^{2}$, Ebru ERSOY ${ }^{3}$, Nuri DANISMAN $^{1}$

\begin{abstract}
A pregnant woman was referred to our perinatal tertiary care center because of fetal bowel distention. Ultrasonography revealed a well-circumscribed circular mass in the fetus' abdomen. After delivery, the male neonate underwent laparotomy to excise the mass. Pathologic examination of the mass was consistent with the diagnosis of fetus in fetu. This case of fetus in fetu was differentiated from a teratoma based on the presence of a vertebral axis and appropriate arrangement of other organs and limbs relative to the vertebral axis. A multidisciplinary clinical approach may be more suitable for managing rare fetal disorders, such as this one.
\end{abstract}

Keywords: Abnormal twinning, enclosed twin, fetus in fetu intraabdominal fetal mass

From: ${ }^{1}$ Department of Perinatology in Zekai Tahir Burak Training and Research Hospital of Women and Children, ${ }^{2}$ Department of Pediatric Surgery, Medical Faculty, Ankara University, ${ }^{3}$ Department of Obstetrics and Gynecology in Zekai Tahir Burak Training and Research Hospital of Women and Children, Ankara, Turkey.

Correspondence: Dr AO Ersoy, Talatpasa Bulv., Hamamonu, 06230, Altindag, Ankara, Turkey. Fax: +903123124931, e-mail: draliersoy@gmail.com 


\section{INTRODUCTION}

Most neonatal abdominal masses are caused by benign retroperitoneal lesions such as hydronephrosis and multicystic dysplastic kidney (1). Fetus in fetu (FiF), also known as an enclosed twin, is a rare condition. It is described as a twin that 'fails to thrive' and is confined in its sibling. It is estimated to affect 1 in 500,000 live births (2). Isaacs reported that FiF was responsible for $25 / 534(4.68 \%)$ of perinatal germ cell tumors (3). The first case was reported by Young in 1809 (4). FiF is distinguished from a teratoma by the presence of a vertebral axis and, commonly, by the appropriate arrangement of other organs and limbs relative to the vertebral axis (5). In $80 \%$ of cases, the FiF was found in the retroperitoneum, while other, atypical sites include: the intracranial area $(6,7)$, mediastinum, lung, oropharynx, scrotum, adrenal gland, ovary, or the neck (8). Because of its malignant potential, the FiF should be completely excised by surgery. The prognosis after resection is usually benign (9).

\section{CASE REPORT}

A 31-year-old multiparous pregnant woman was referred to our perinatal tertiary care center after a routine ultrasound scan showed that her 37-week-old fetus had bowel distention. The woman was in a non-consanguineous marriage and had no significant medical or gestational history. There was no history of twin gestation. Detailed sonogram and follow-up ultrasound of the index pregnancy revealed no notable clinical signs.

A senior obstetrician examined the patient via ultrasound, and noted that the fetal biometry was consistent with a gestational age of 34-35 weeks, there was severe oligohydramnios. There was a well-circumscribed circular mass measuring $44 \times 38 \times 40 \mathrm{~mm}$ with echoes consistent with calcification. The mass was located in the central upper abdomen, below the liver, in front of and between two fetal renal pelvises (Figure 1A). 
The male neonate was delivered via Caesarean section at 37 weeks and 1 day of gestation, because of fetal distress. The birth weight was $2740 \mathrm{~g}$. The Apgar scores were 7 at $1 \mathrm{~min}$ and 9 at $5 \mathrm{~min}$. The mother was discharged 2 days after delivery, with no remarkable medical concerns at this time.

A physical examination of the neonate revealed that the only notable sign was mild to moderate abdominal distention. The neonate's complete blood count and arterial blood gas were normal. Ultrasound confirmed that the locations and structures of the liver, gall bladder, spleen, and suprarenal glands were normal. The mass in the neonate's abdomen had the same features as those observed in the prenatal examination. Respiration and alimentation were noted as being normal. An experienced pediatric cardiologist reported that the neonate's cardiac system was normal. A computed tomography scan confirmed the ultrasound and physical examination findings (Figure 1B).

The pediatric surgical team decided to perform a laparotomy to excise the mass. In open view during surgery, the mass compressed the duodenum from the posterior side (Figure 1C). After opening the retroperitoneal space, it was possible to see the hemorrhagic structure of the mass (Figure 1D). Excision of the mass eliminated the compression on the duodenum (Figure 1E).

The mass was removed intact and sent for pathologic and genetic examination. The mass weighed $220 \mathrm{~g}$ and measured $40 \times 30 \times 25 \mathrm{~mm}$. A radiograph of the mass showed linear calcification and the presence of a vertebral column and limbs (Figure 1F). Pathologic and macroscopic examination of the mass revealed its hemorrhagic structure, including the presence of fatty tissue, irregularly arranged bone, cartilage, and a skin-covered conglomeration of recognizable rudimentary fetal parts (Figure 1G). In addition, a rudimentary axial skeleton was found with 3 rudimentary limbs; the largest limb was $15 \mathrm{~mm}$ long and the smallest limb was $10 \mathrm{~mm}$ long. Microscopic examination confirmed that the 
mass was covered with skin and contained some osseous tissue and a rudimentary gastrointestinal tract. The final pathologic report stated that the mass was consistent with an acephalic acardiac fetus. Cytogenetic examination of the mass revealed that the karyotyping was $46 \mathrm{XY}$.

The neonate has experienced no complications at the last follow-up, 6 months after surgery.

\section{DISCUSSION}

This case of FiF was differentiated from a teratoma by the presence of a vertebral axis and appropriate arrangement of other organs and limbs relative to the vertebral axis (5). The FiF also had a similar appearance to other cases reported in the literature.

Most authors have attributed the etiology of FiF to anomalous cleavage of monozygotic twins, where one fetus fails to thrive and is entrapped by the other fetus when the anterior abdominal wall closes around the umbilicus and linea alba (10). Therefore, the FiF must be entrapped during the second half of the first trimester or in the early phase of the second trimester.

Although FiF is more common in females (11), the gender of our neonate was male. The FiF was localized in the retroperitoneal space, the most commonly reported site.

Prenatal diagnosis of this rare condition may be difficult because of other possible diagnoses, including hydronephrosis, cystic abnormalities, solid tumors of the kidney, intestinal malformations, teratoma, choledochal cyst, neuroblastoma, adrenal hemorrhage, and infra-diaphragmatic pulmonary sequestration (12). Ultrasound in the second trimester did not reveal a possible intraabdominal mass in the present case, as in an earlier case reported by Huddle et al (7). Therefore, FiF might not be visible until late pregnancy. 
Most clinicians agree on the superiority of ultrasound imaging in everyday clinical practice, but it may be unsatisfactory in some circumstances. In the management of this case, it is possible that antepartum magnetic resonance imaging could have aided the diagnosis of FiF antenatally, or allowed us to exclude other types of intraabdominal fetal mass from the differential diagnosis, if it had been performed. Nevertheless, pathologic examination is essential to reach the final diagnosis in such cases because all screening and imaging methods have some limitations that could hinder accurate diagnosis.

Huddle et al. highlighted the need for further genetic investigations, such as microarray or single nucleotide polymorphism analyses (7). It may be valuable to use these tools beyond conventional cytogenetic analysis because they can provide additional information regarding the identity of twins. Nevertheless, we think that decision to use these tools must be clinically justified.

In conclusion, $\mathrm{FiF}$ is a rare clinical condition and is seldom considered in the differential diagnosis of a fetal intraabdominal mass. If a clinician suspects a fetal abdominal mass, he/she should contact expert perinatologists and pediatric surgeons. Wherever possible, the fetus should be delivered in a clinic with suitable facilities, including an expert obstetric team, pediatric surgery, and neonatal intensive care unit. A multidisciplinary clinical approach may be more suitable for the management of such rare cases.

Declaration of interest: The authors report no conflicts of interest. The authors alone are responsible for the content and writing of the paper. 


\section{REFERENCES}

1. Hartman GE, Shochat SJ. Abdominal mass lesions in the newborn: diagnosis and treatment. Clin Perinatol 1989; 16: 123-35.

2. Grant P, Pearn JH. "Foetus-in-foetu". Med J Aust 1969; 1: 1016-9.

3. Isaacs H Jr. Perinatal (fetal and neonatal) germ cell tumors. J Pediatr Surg 2004; 39: 1003-13.

4. Young GW. Case of a foetus found in the abdomen of a boy. Med Chir Trans1809;1: 234-62.

5. Willis RA. The borderline of embryology and pathology. In: The embryonic tumours and teratomas. London, UK: Butterworth and Co., 1958; 147.

6. Goldstein I, Jakobi P, Groisman G, Itskovitz-Eldor J. Intracranial fetus-in-fetu. Am J Obstet Gynecol 1996; 175: 1389-90.

7. Huddle LN, Fuller C, Powell T, Hiemenga JA, Yan J, Deuell B, et al. Intraventricular twin fetuses in fetu. Neurosurg Pediatrics 2012; 9: 17-23.

8. Tofigh AM, Kavyani A, Abdollahi SM, Kazemzadeh G, Salman DN. Fetus in fetu: report of a case and literature review. Int J Surg 2008; 6: e94-e96.

9. Khadaroo RG, Evans MG, Honore LH, Bhargava R, Phillipos E. Fetus in fetu presenting as cystic meconium peritonitis: diagnosis, pathology, and surgical management. J Pediatr Surg 2000; 35: 721-3.

10. Hoeffel C, Khoang Q, Tran T, Fornes P. Fetus in fetu: a case report and literature review. Pediatrics 2000; 105: 1335-44.

11. Spencer R. Parasitic conjoined twins: external and internal (fetus in fetu and teratomas), and detached (acardiacs). Clin Anat 2001; 14: 428-44.

12. Chandler JC, Gauderer MWL. The neonate with an abdominal mass. Pediatr Clin N Am 2004; 51: 979 -997. 


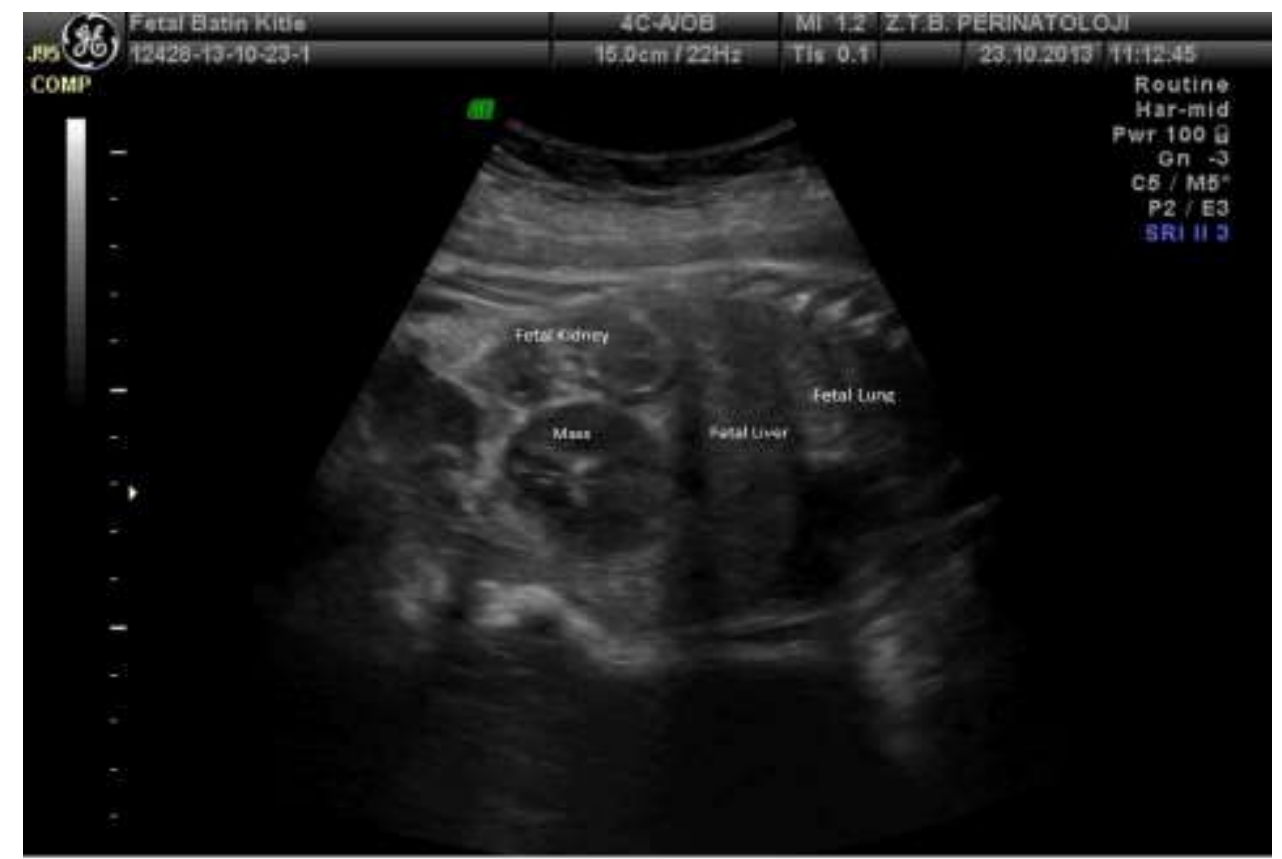

Fig 1A. Coronal plane ultrasound view of the mass located in the fetal abdomen.

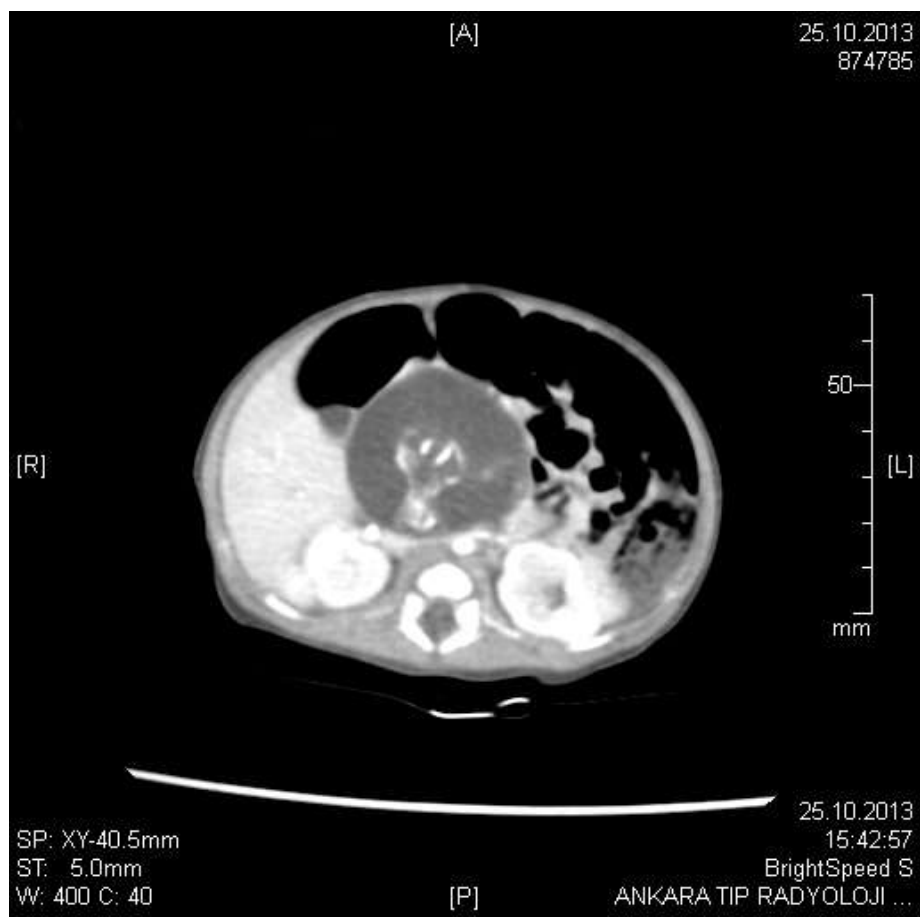

Fig 1B. Computed tomographic (CT) view of the neonate shows the mass in the central abdomen._This view also shows the kidneys, liver, and mildly dilated bowel of the neonate. 


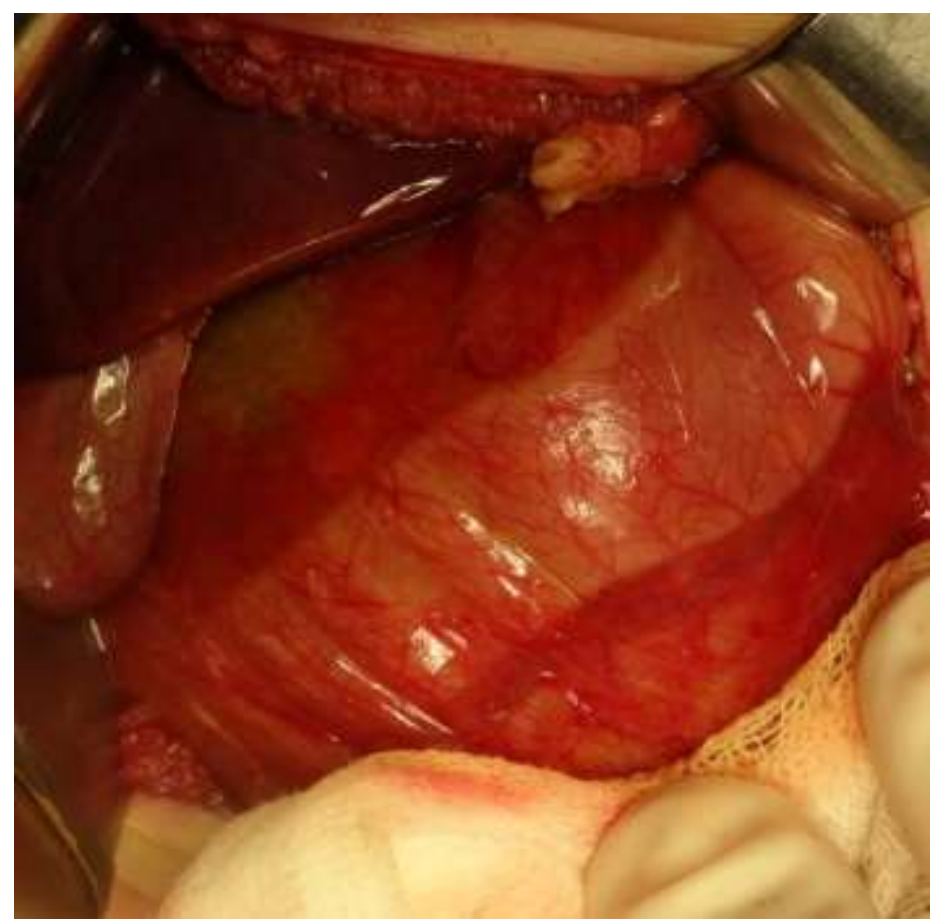

Fig 1C. An open view of the neonate's liver, gall bladder and duodenum. The mass compresses the duodenum from the posterior side.

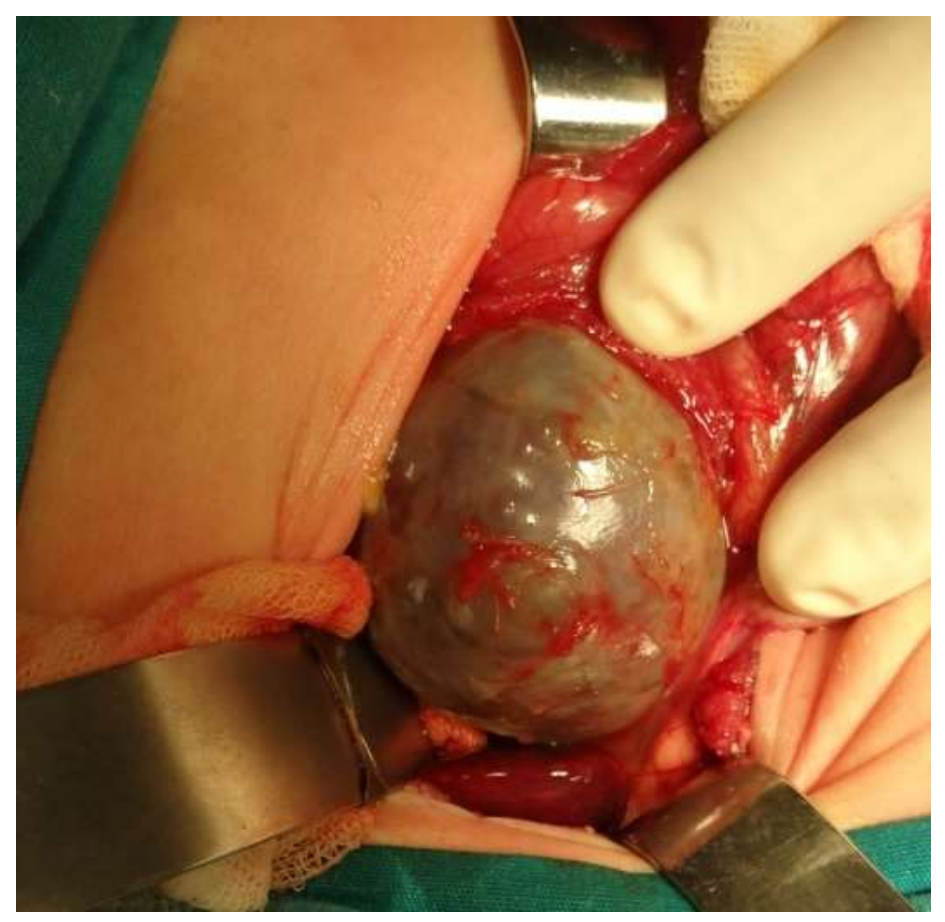

Fig 1D. Image of the mass in the retroperitoneal space showing its congestive and hemorrhagic structure. 


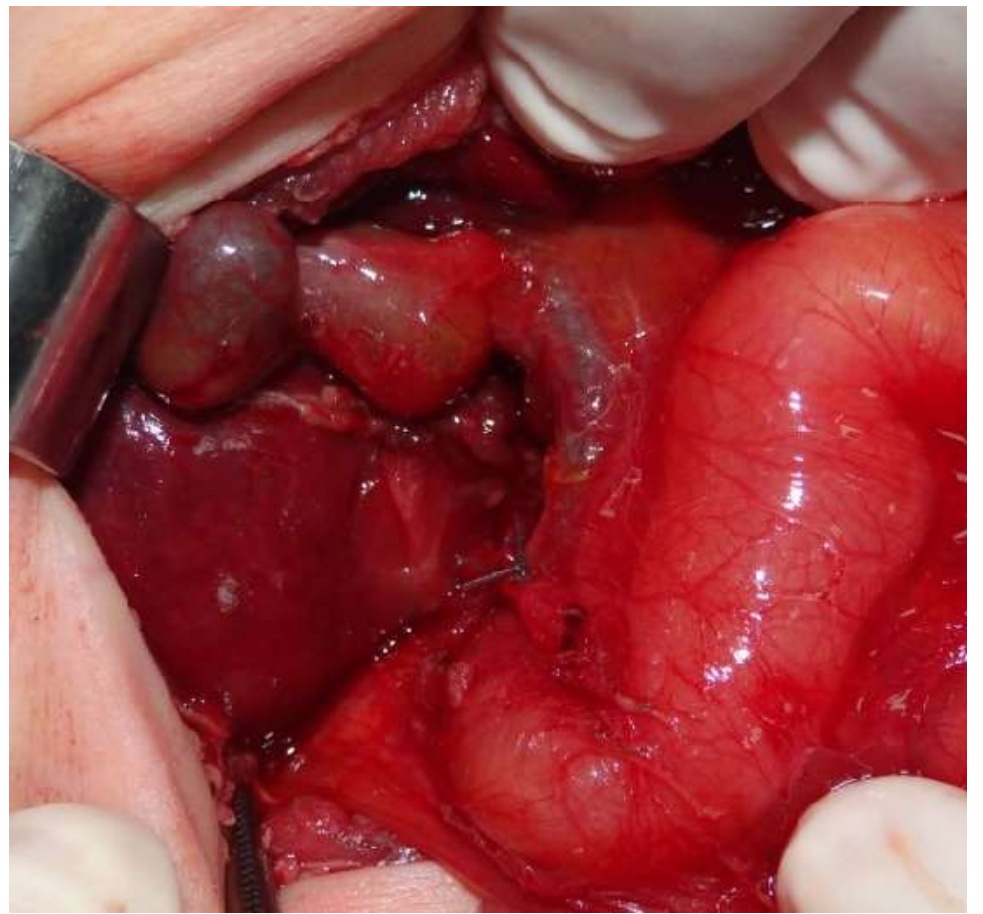

Figure 1E. Excision of the mass eliminated the compression on the duodenum

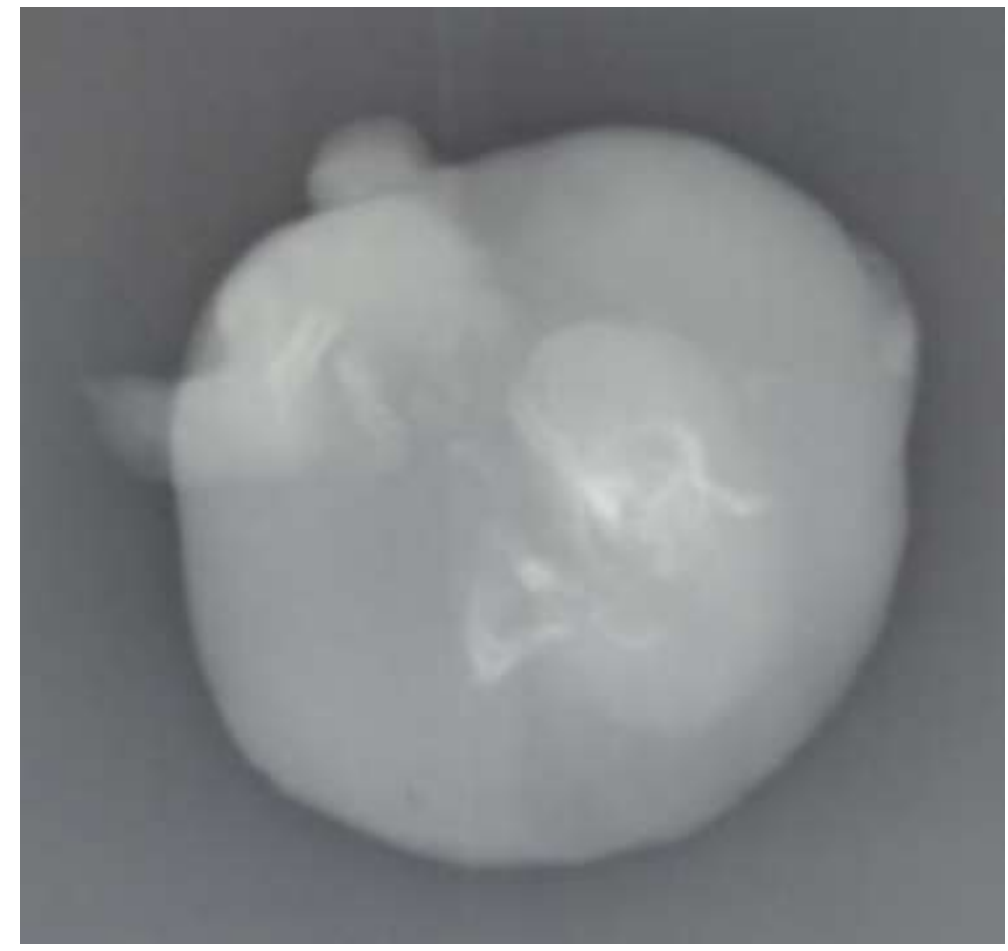

Fig 1F. X-ray image of the mass. 


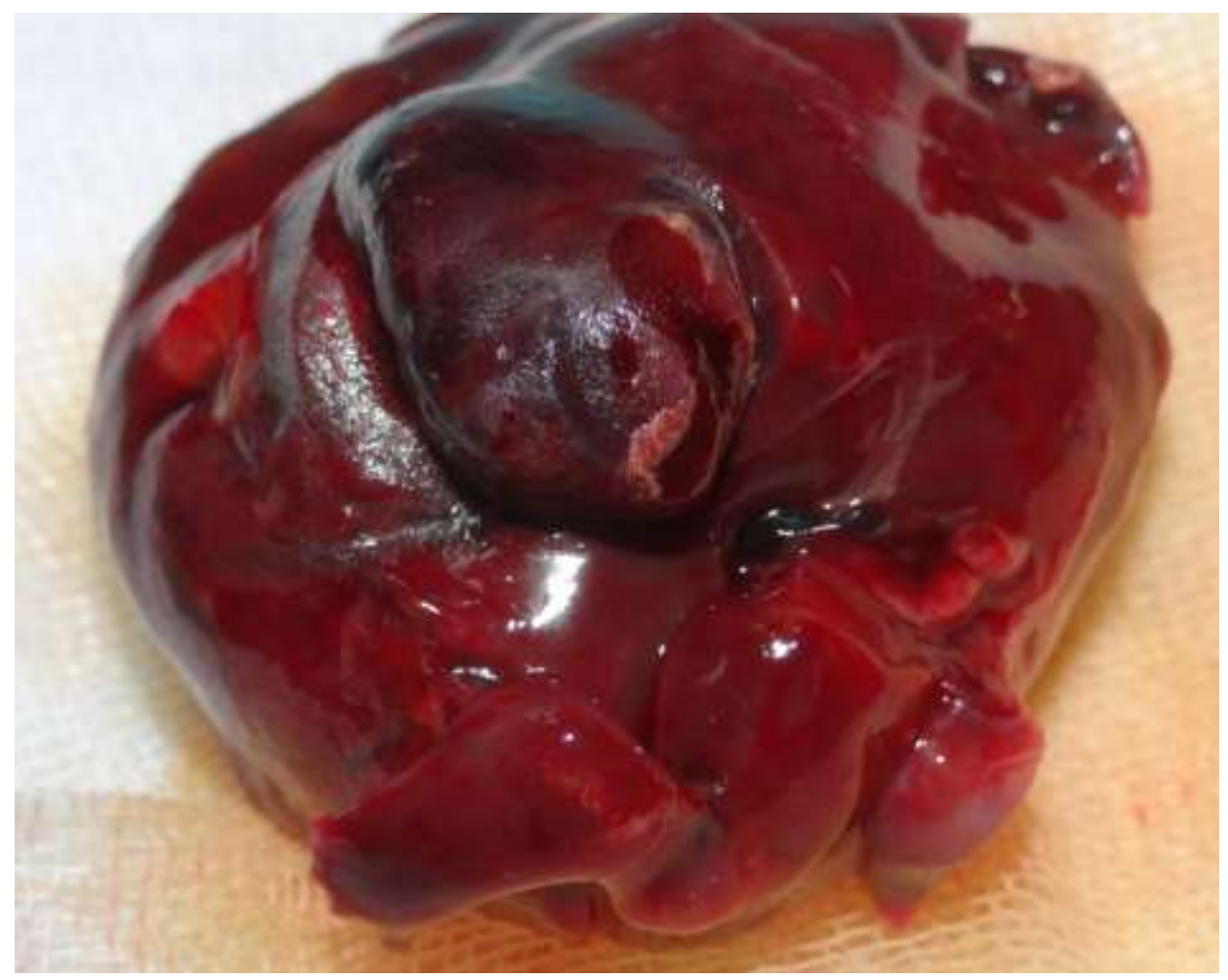

Fig 1G. Internal view of the mass after removing its outer membrane. Structures consistent with limbs, a face, and a gastrointestinal tract can be seen. 